\title{
Morphological Control of Periodic GaAs Hole Arrays by Simple Au-Mediated Wet Etching
}

Yukiko Yasukawa, Hidetaka Asoh and Sachiko Ono

J. Electrochem. Soc. 2012, Volume 159, Issue 5, Pages D328-D332. doi: 10.1149/2.021206jes $\begin{array}{cl}\begin{array}{c}\text { Email alerting } \\ \text { service }\end{array} & \begin{array}{l}\text { Receive free email alerts when new articles cite this article - sign up } \\ \text { in the box at the top right corner of the article or click here }\end{array}\end{array}$

To subscribe to Journal of The Electrochemical Society go to: http://jes.ecsdl.org/subscriptions 


\title{
Morphological Control of Periodic GaAs Hole Arrays by Simple Au-Mediated Wet Etching
}

\author{
Yukiko Yasukawa, ${ }^{\text {a,c }}$ Hidetaka Asoh, ${ }^{\text {a,b }}$ and Sachiko Ono ${ }^{\text {a,b,*,z }}$ \\ ${ }^{a}$ Research Institute for Science and Technology, Kogakuin University, Hachioji, Tokyo 192-0015, Japan \\ ${ }^{b}$ Department of Applied Chemistry, Faculty of Engineering, Kogakuin University, Hachioji, Tokyo 192-0015, Japan
}

\begin{abstract}
In this study we report etched-GaAs morphologies with noble-metal layers embedded in the etching structures that are prepared by a simple wet process. Well-controlled n-GaAs (100) hole arrays are formed through metal-assisted chemical etching using a sputtered Au layer as an etching catalyst. GaAs exhibits anisotropic etching behavior, which originates from the substrate crystallography. The configuration of hole arrays is determined by the concentration of hydrofluoric acid included in the etching solution, the arrangement of Au catalysis layers relative to the preferential etching direction of the GaAs (100) substrate, and the etching time. The relationship between the etching process and the resultant hole structure is also discussed.

C 2012 The Electrochemical Society. [DOI: 10.1149/2.021206jes] All rights reserved.
\end{abstract}

Manuscript submitted December 26, 2011; revised manuscript received February 28, 2012. Published March 19, 2012.

Low-dimensional materials such as porous silicon $(\mathrm{Si}),{ }^{1} \mathrm{Si}$ nanowires, ${ }^{2}$ carbon nanotubes $(\mathrm{CNTs}),{ }^{3}$ and titanium dioxide $\left(\mathrm{TiO}_{2}\right)$ nanotubes ${ }^{4}$ have attracted considerable attention because of their modified properties, which originate from their size effects. Canham ${ }^{5}$ and Lehmann and Gösele ${ }^{6}$ discovered the visible-light-emitting property and quantum size effect of electrochemically prepared porous $\mathrm{Si}$ in the early 1990s. Since then, the high potential of porous semiconductors has been recognized and there has been intense research on porous $\mathrm{Si}$ and also porous III-V semiconductors. ${ }^{7-9}$ Concerning porous GaAs, applications to solar cells and optoelectronic devices can be expected. For instance, p-type GaAs with nanometer-scale pores exhibits yellow and infrared photoluminescence bands owing to the quantum effect. ${ }^{10}$

$\mathrm{Li}$ and Bohn reported metal-assisted chemical etching, ${ }^{11}$ which is one of the simplest processes for fabricating porous $\mathrm{Si}$ without the use of an electric power source. In their study, a thin noble-metal layer on a Si substrate played an important role as an etching catalyst. They demonstrated that porous $\mathrm{Si}$ is formed by immersing substrates that have been decorated by noble-metal layers in an etching solution consisting of hydrofluoric acid (HF) and hydrogen peroxide $\left(\mathrm{H}_{2} \mathrm{O}_{2}\right)$. In metal-assisted chemical etching, an interface reaction between $\mathrm{Si}$ and the noble metal occurs, thereby the oxidative dissolution of Si takes place, leading to the formation of a porous Si layer. Moreover, metalassisted chemical etching was also realized on a Pd-deposited polycrystalline $\mathrm{Si}$ wafer using $\mathrm{HF}$ and sodium peroxydisulfate $\left(\mathrm{Na}_{2} \mathrm{~S}_{2} \mathrm{O}_{8}\right)$ solutions, resulting in the formation of porous Si layers. ${ }^{12}$ In Ref. 12, a significant decrease in the reflectance of the porous layers was found, indicating their potential application to solar cells.

Polystyrene (PS) spheres form a self-organized structure, and such a structure was first used as a lithographic mask for the surface patterning of Si in 1982 by Deckman and Dunsmuir. ${ }^{13}$ They obtained various sizes of microcolumnar $\mathrm{Si}$, which revealed the size tunability of the mask by varying the diameter of the PS spheres. Since then, the versatility and usefulness of masks made from self-organized PS spheres for the patterning of flat substrates have become widely recognized. For instance, Si macropore arrays ${ }^{14}$ and self-organized $\mathrm{Au}$ dot arrays on Si substrates ${ }^{15}$ were successfully fabricated using such masks.

With this background, we have attempted to obtain n-type GaAs hole arrays through metal-assisted chemical etching using a selforganized colloidal crystal mask consisting of PS and silica $\left(\mathrm{SiO}_{2}\right)$ spheres. $\mathrm{Ag}^{16}$ and $\mathrm{Pt}-\mathrm{Pd}^{17}$ were used as etching catalysts for the fabrication of hole arrays, but it was hard to control the etching itself by using these metals as catalysts in our previous studies. Herein, we attempt to realize more precise control in the fabrication of GaAs hole

${ }^{*}$ Electrochemical Society Active Member.

${ }^{c}$ Present address: Department of Information Engineering, Faculty of Engineering, Shinshu University, Nagano 380-8553, Japan.

${ }^{\mathrm{z}}$ E-mail: sachiono@cc.kogakuin.ac.jp arrays by using a sputtered $\mathrm{Au}$ layer as a catalyst for metal-assisted chemical etching.

\section{Experimental}

In the present study, a Si-doped GaAs (100) wafer with a resistivity of $10^{-3} \Omega \mathrm{cm}$ order was used as a substrate. The fabrication process of hole arrays is exhibited in Figs. 1a-1e. A solution containing $\mathrm{SiO}_{2}$ $(\phi 3 \mu \mathrm{m})$ and PS $(\phi 0.2 \mu \mathrm{m})$ spheres was used as the source of the etching mask formed on the substrate. ${ }^{17,18}$ After the evaporation of the solvent, the $\mathrm{SiO}_{2}$ spheres formed a self-organized structure filled with the smaller PS spheres in the interstitial spaces (Fig. 1a). For the fixation and agglomeration of neighboring PS spheres, the substrate was heated at $105^{\circ} \mathrm{C}$ for $1 \mathrm{~h}$. The substrate was then immersed in $10 \mathrm{wt} \% \mathrm{HF}$ for $10 \mathrm{~min}$ for the selective dissolution of the $\mathrm{SiO}_{2}$ spheres (Fig. 1b). The remaining PS spheres on the substrate formed a honeycomb structure, resulting in a PS-honeycomb structural mask. Afterward, Au was sputtered (Hitachi E-1010) as a noble-metal catalyst on the substrate through the mask (Fig. 1c). The thickness of the $\mathrm{Au}$ layer was adjusted by varying the intensity of the discharge current and sputtering time to achieve a thickness of $\sim 20 \mathrm{~nm}$. By soaking the specimen in toluene and then acetone while subjecting it to ultrasonic waves only the PS-honeycomb structural mask was removed and the $\mathrm{Au}$ layer was remained on the substrate. Thus, islandlike $\mathrm{Au}$ layers were formed at sites where $\mathrm{SiO}_{2}$ spheres were previously located (Fig. 1d). Lastly, the substrate with patterned Au layers was immersed in an etching solution consisting of 3-5 mol dm ${ }^{-3} \mathrm{HF}$ and $1 \mathrm{~mol} \mathrm{dm}^{-3} \mathrm{H}_{2} \mathrm{O}_{2}$ for various etching times (Fig. 1e). The resultant etching structures were observed by field-emission scanning electron microscopy (FE-SEM, Hitachi S-4200) and atomic force microscopy (AFM, Digital Instruments NanoScope IIIa).

\section{Results and Discussion}

Effect of substrate crystallography on hole structures.- Through the PS honeycomb mask, the Au layer was deposited by sputtering at sites where self-organized $\mathrm{SiO}_{2}$ spheres had been removed. The obtained islandlike Au pattern on the substrate, which was used as an etching catalyst, was a hexagonal close-packed array. Each Au layer was circular in accordance with the contact areas between the $\mathrm{SiO}_{2}$ spheres and the substrate. After deposition, the specimen was immersed in the etchant composed of $3 \mathrm{~mol} \mathrm{dm}^{-3} \mathrm{HF}$ and $1 \mathrm{~mol} \mathrm{dm}^{-3}$ $\mathrm{H}_{2} \mathrm{O}_{2}$ for 90 s. Figures $2 \mathrm{a}-2 \mathrm{~d}$ show SEM images of the GaAs hole arrays fabricated under the above conditions. In a previous study, we obtained hole arrays with circular apertures by Pt-Pd-assisted chemical etching under similar etching conditions to those in the present study. ${ }^{17}$ The rectangular holes obtained in this study (Figs. 2a-2d) are attributed to a combination of Au-assisted chemical etching with a lower etching rate than that in the case of Pt-Pd and the anisotropic 
a

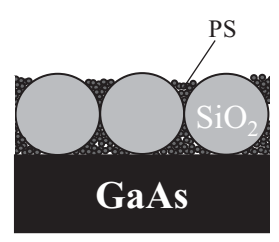

d

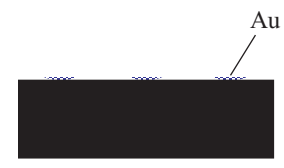

b
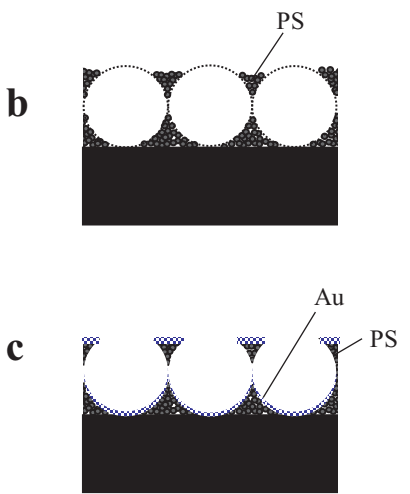

Figure 1. (Color online) Formation of GaAs hole arrays. (a) Colloidal crystals consisting of self-organized large $\mathrm{SiO}_{2}$ and small PS spheres, which were fixed on the substrate by heat-treatment. (b) Selective dissolution of $\mathrm{SiO}_{2}$ spheres and resultant PS honeycomb-structure mask. (c) Au layer sputtered through the PS mask. (d) Removal of the mask maintaining the Au layer on the substrate. (e) Hole arrays after Au-assisted chemical etching.
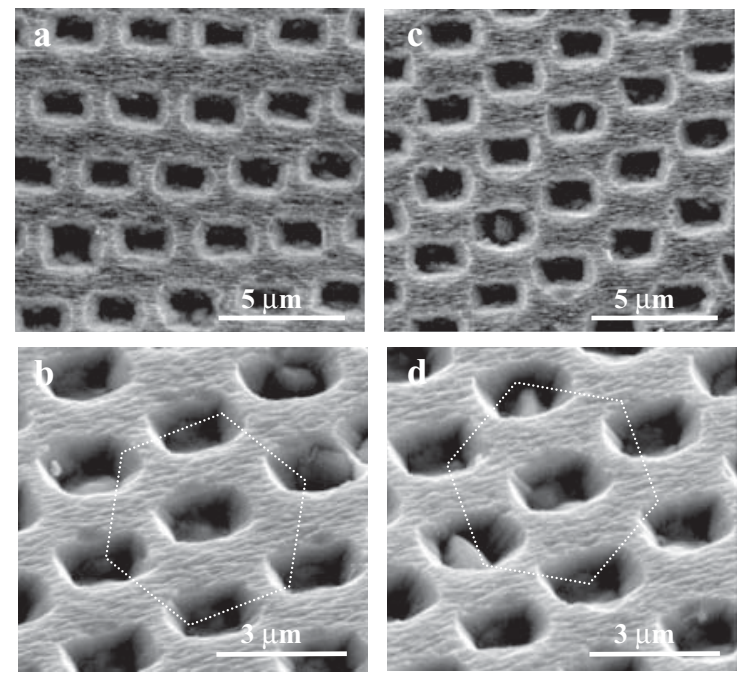

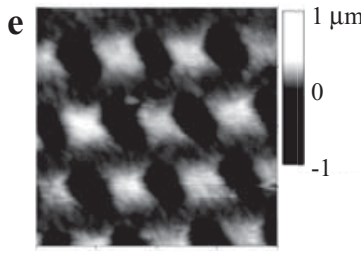

$\mathrm{X}, \mathrm{Y}: 2 \mu \mathrm{m} / \mathrm{div}$

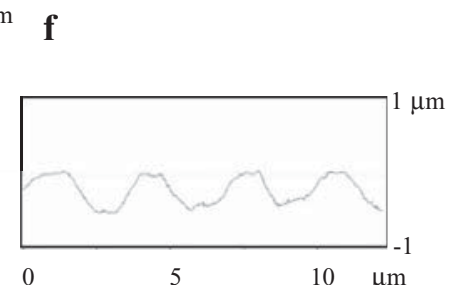

0

$10 \mu \mathrm{m}$
Figure 2. (Color online) GaAs hole arrays formed by Au-assisted chemical etching for $90 \mathrm{~s}$ in $3 \mathrm{~mol} \mathrm{dm}^{-3} \mathrm{HF}$ and $1 \mathrm{~mol} \mathrm{dm}^{-3} \mathrm{H}_{2} \mathrm{O}_{2}$. Top-view SEM images (a) and (c) and corresponding SEM images taken at $45^{\circ}$ to the surface (b) and (d). (e) Surface morphology measured by AFM and (f) corresponding line profile. etching of n-GaAs (100). On the basis of our empirical results, we revealed that $\mathrm{Pt}-\mathrm{Pd}$ has a higher etching rate than $\mathrm{Au}, \mathrm{Pd}$, and $\mathrm{Ag}$ in the metal-assisted chemical etching of $n-G a A s$. The catalytic ability of these metals, which affects the etching rate, will be compared later.

The wider and narrower apertures of the rectangular holes correspond to the lateral and vertical directions in the SEM images, respectively. The sides of the rectangles appearing in the images are the most crystallographically stable facets. Preferential etching, which corresponds to a higher etch rate, can be recognized to occur in the lateral direction in Figs. 2a, 2b and 2c, 2d. Conversely, the vertical direction of the images is identified as being a less preferential etching direction. The close-packed arrangements of the hexagonally ordered holes are different between Figs. $2 \mathrm{a}, 2 \mathrm{~b}$ and $2 \mathrm{c}, 2 \mathrm{~d}$ as indicated by the dashed hexagons. This matter will also be discussed later. Note that the Au layer before etching was circular but arrays of holes with rectangular apertures were formed through etching. The results clarified that the morphological control of etching structures in metal-assisted chemical etching, such as from structures with circular apertures to those with rectangular apertures is realized by changing the species of etching catalyst from Pt-Pd to Au. Namely, the adjustment of the etching rate by altering the species of catalyst enabled us to prepare various surface morphologies.

The surface morphology and etching depth of the hole arrays corresponding to Figs. $2 \mathrm{a}-2 \mathrm{~d}$ were evaluated by AFM measurements, the results of which are shown in Figs. 2e and 2f. The etching widths of the holes were $\sim 1.5 \mu \mathrm{m}$ and $\sim 1.2 \mu \mathrm{m}$ in the preferential and less preferential etching directions, respectively. Therefore, the difference in the hole aperture width between the faster and slower etching directions is $\sim 0.3 \mu \mathrm{m}$, corresponding to a difference in the etching rate of $3.3 \mathrm{~nm} \mathrm{~s}^{-1}$. The average depth of holes in the direction perpendicular to the substrate, i.e., the [100] direction, was approximately $0.7 \mu \mathrm{m}$; thus, the calculated etching rate in the [100] direction is $\sim 7.8 \mathrm{~nm} \mathrm{~s}^{-1}$.

Precipitation of catalytic metal layers in hole arrays.- Cross sections of GaAs hole arrays prepared in this study are shown in Figs. $3 \mathrm{a}$ and $3 \mathrm{~b}$ (etched in $5 \mathrm{~mol} \mathrm{dm}{ }^{-3} \mathrm{HF} / 1 \mathrm{~mol} \mathrm{dm}^{-3} \mathrm{H}_{2} \mathrm{O}_{2}$ for $60 \mathrm{~s}$ ). As observed in the images, each hole was tapered with a greater width
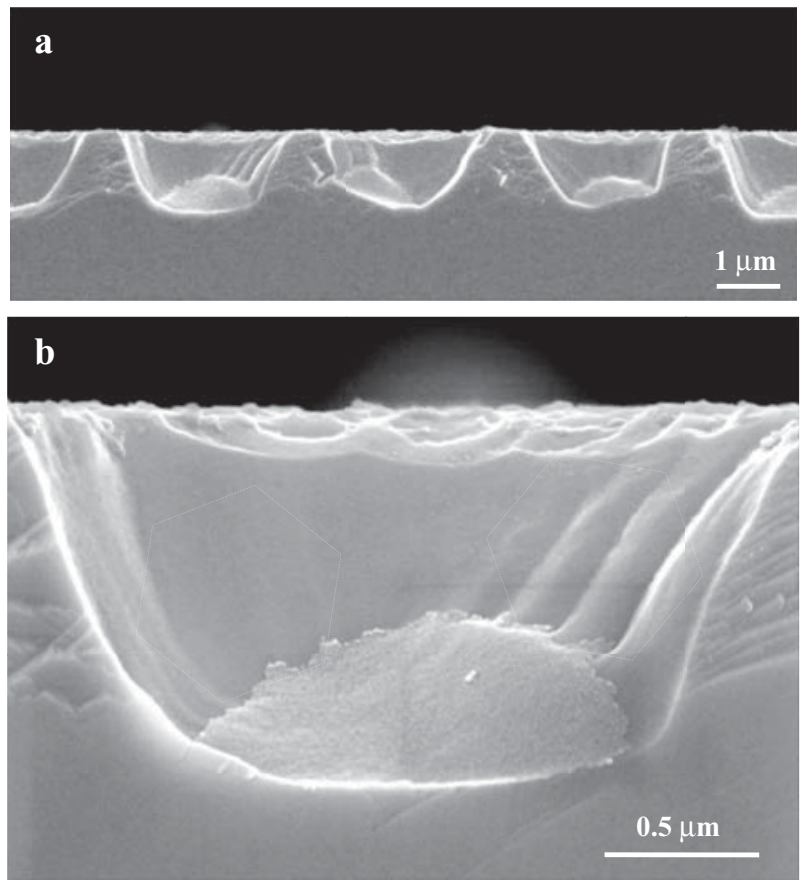

Figure 3. Cross sections of hole arrays (a) and representative enlarged image (b). Holes were obtained in an etchant of $5 \mathrm{~mol} \mathrm{dm}{ }^{-3} \mathrm{HF} / 1 \mathrm{~mol} \mathrm{dm}{ }^{-3} \mathrm{H}_{2} \mathrm{O}_{2}$ for $60 \mathrm{~s}$. 
a

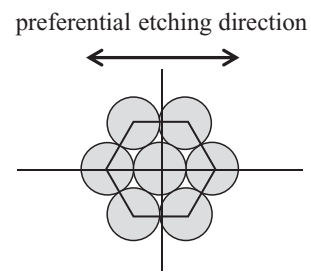

b

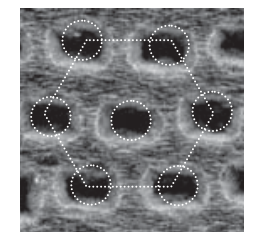

c

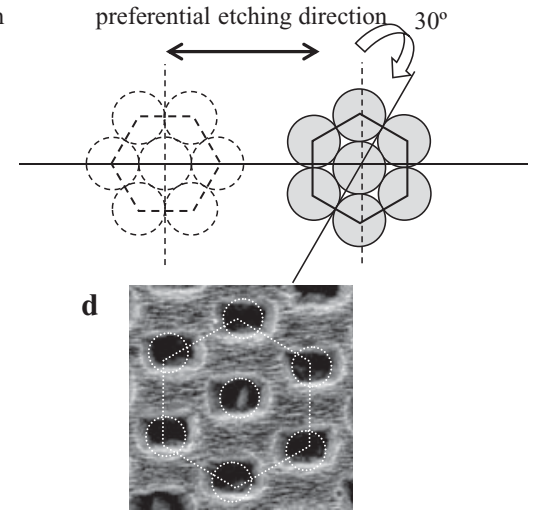

Figure 4. Relationship between the crystallographic direction of GaAs and the arrangement of holes. (a) and (c) Arrangements of islandlike Au layers exhibiting different ordering. In (a), the ordering of the Au layer is parallel to the preferential etching direction, while in (c) it is oriented $30^{\circ}$ to the preferential direction. (b) and (d) SEM images.

at the top and a narrower width at the bottom. The top width was $\sim 2.5 \mu \mathrm{m}$, while the bottom width was $\sim 1.5 \mu \mathrm{m}$ with an etching depth of $\sim 1.2 \mu \mathrm{m}$. One of the notable features of the etching structure is the precipitation of a film-shaped Au layer used as the etching catalyst at the bottom of each hole. Although metal-assisted chemical etching proceeded in the radial direction, the results indicate that the etching rate is highest at the interface between GaAs and Au. Therefore, [100] is the dominant etching direction.

Arrangement of hole arrays in relation to crystallographic direction.- The relationship between the crystallographic direction of the GaAs substrate and the arrangement of holes is schematically shown in Fig. 4. In Figs. $4 b$ and $4 d$, the etching traces of the substrate were found to align with the lateral direction for both images. Therefore, the preferential etching direction of the GaAs (100) substrate is

parallel to the lateral direction of the images as indicated by arrows. By considering the arrangements of holes and etching traces of the substrate, it is possible to deduce the angle of hole arrangements concerning the preferential etching direction. Based on these perspectives, it is reasonable to define the arrangements of holes to be 0 degree in relation to the preferential etching direction when the minimum unit of the hexagonal close-packed Au layer is aligned with parallel to the preferential etching direction (Figs. $4 \mathrm{a}$ and $4 \mathrm{~b}$ ). The locations of $\mathrm{Au}$ layers before etching are indicated as dotted circles in Fig. 4b, whereas the hexagonal dotted line represents the self-organized unit. In this situation, holes are alternately ordered layer by layer, i.e., holes are alternately stacked in the perpendicular direction in Fig. $4 \mathrm{~b}$ to form a close-packed arrangement. Note that this also occurs when the selforganized Au layers are oriented at $60^{\circ}$ relative to the preferential etching direction. On the other hand, the arrangement of Au layer is oriented at $30^{\circ}$ to the preferential etching direction (Figs. $4 \mathrm{c}$ and $4 \mathrm{~d}$ ) by considering the traces of substrate etching and hole arrangements. In this case, the holes are formed alternately line by line, i.e., the holes are aligned in the vertical direction in Fig. 4d. It was concluded that two types of hole arrangement are possible by changing the arrangement of the Au layer relative to the preferential etching direction of the GaAs (100) substrate.

Extra degree of freedom in etching morphologies.- The effect of etching time on the morphology is shown in Figs. 5a-5f for the cases of parallel and $30^{\circ}$-oriented hole arrangements relative to the preferential etching direction. The lateral direction represents the preferential etching direction in all the images and schematics. In Figs. 5a-5c, the arrangement of holes is parallel to the preferential direction. Specimens were obtained by Au-assisted chemical etching in $3 \mathrm{~mol} \mathrm{dm}^{-3}$ $\mathrm{HF} / 1 \mathrm{~mol} \mathrm{dm}^{-3} \mathrm{H}_{2} \mathrm{O}_{2}$ for $90 \mathrm{~s}$ (Fig. 5a) and $5 \mathrm{~mol} \mathrm{dm}{ }^{-3} \mathrm{HF} / 1 \mathrm{~mol}$ $\mathrm{dm}^{-3} \mathrm{H}_{2} \mathrm{O}_{2}$ for $120 \mathrm{~s}$ (Fig. 5b). As Au-assisted chemical etching proceeds, the hole apertures became wider in both the preferential and less preferential directions (Figs. 5c-1 and 5c-2). The apertures became increasingly rectangular (Fig. 5c-3) with increasing etching depth as the etching time increased. Au-assisted chemical etching was also conducted using $5 \mathrm{~mol} \mathrm{dm}{ }^{-3} \mathrm{HF} / 1 \mathrm{~mol} \mathrm{dm}^{-3} \mathrm{H}_{2} \mathrm{O}_{2}$ for $60 \mathrm{~s}$ (Fig. 5d) and $180 \mathrm{~s}$ (Fig. 5e). In these cases, the holes were oriented $30^{\circ}$ relative to the preferential etching direction. The holes in Fig. $5 \mathrm{~d}$
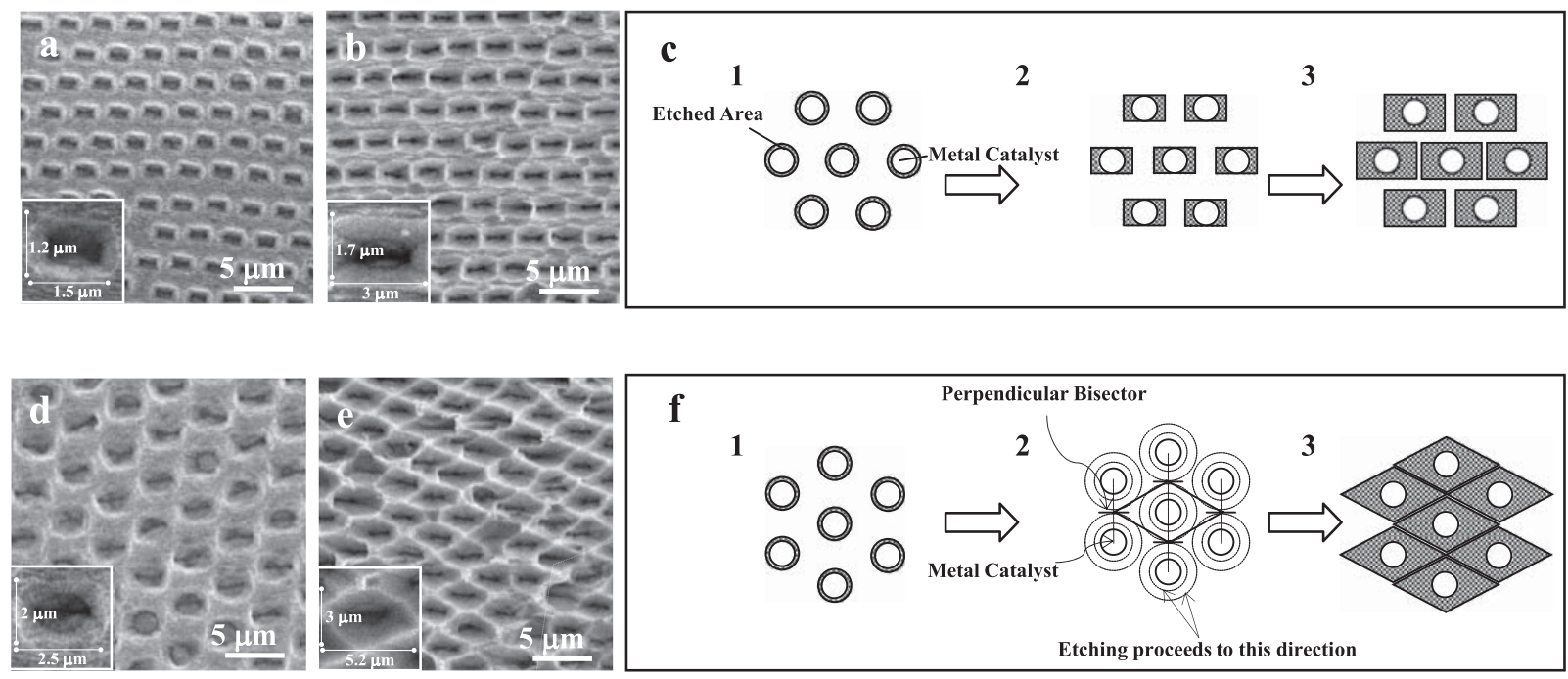

Figure 5. Effect of etching time on morphology for different arrangements of holes (a)-(c) and (d)-(f). Top-view SEM images of arrangements after Au-assisted chemical etching for (a) $90 \mathrm{~s}$ in a mixture of $3 \mathrm{~mol} \mathrm{dm}-3 \mathrm{HF}$ and $1 \mathrm{~mol} \mathrm{dm}^{-3} \mathrm{H}_{2} \mathrm{O}_{2}$ and (b) $120 \mathrm{~s}$ in a mixture of $5 \mathrm{~mol} \mathrm{dm}^{-3} \mathrm{HF}^{-1} 1 \mathrm{~mol} \mathrm{dm}^{-3} \mathrm{H}_{2} \mathrm{O}_{2}$. (c) Schematic etching mechanisms as a function of etching time (1)-(3) for (a) and (b). (d) and (e) Top views after etching for $60 \mathrm{~s}$ and $180 \mathrm{~s}$ in $5 \mathrm{~mol} \mathrm{dm}^{-3} \mathrm{HF} / 1$ mol dm ${ }^{-3} \mathrm{H}_{2} \mathrm{O}_{2}$, respectively. The etching processes in (d) and (e) are schematically shown in (f), (1)-(3). 
are essentially rectangular but are somewhat elliptical compared with those in Fig. 5a. This could be attributed to the higher HF concentration in the etchant, which induces a higher etching rate, although the etching time of the specimen shown in Fig. 5d is shorter than that of the specimen in Fig. 5a. Generally, GaAs forms a Ga complex in HF solution. Subsequently, the GaAs substrate is chemically dissolved owing to the formation of the complex. The reaction leading to the formation of the complex and the following chemical dissolution of the substrate should be enhanced with increasing HF concentration. ${ }^{17}$ In consequence, the apertures underwent greater dissolution in the etchant with a higher HF concentration, which led to the elliptical holes.

When the etching time was increased threefold from 60s (Fig. 5d) to $180 \mathrm{~s}$ (Fig. 5e), the holes became parallelogram-shaped. We consider that the parallelogram-shaped holes originated as schematically shown in Figs. $5 \mathrm{f}-1$ to $5 \mathrm{f}-3$. Relative to an islandlike Au layer, metalassisted chemical etching proceeds in the radial direction (Figs. 5f-1 and 5f-2). Similarly, radial etching takes place in neighboring islandlike Au layers as shown by dotted circles (Fig. 5f-2). As a result, the perpendicular bisector between the centers of neighboring Au layers in Fig. $5 \mathrm{f}-2$ becomes the boundary between hole structures, resulting in the formation of parallelogram-shaped holes (Fig. 5f-3).

After Au-assisted chemical etching, the dimensions of the hole apertures in the preferential and less preferential etching directions according to the inserted SEM images were $1.5 \mu \mathrm{m}$ and $1.2 \mu \mathrm{m}$ in Fig. 5a and $2.5 \mu \mathrm{m}$ and $2 \mu \mathrm{m}$ in Fig. 5d, giving aspect ratios of 1.25 , whereas the dimensions were $3 \mu \mathrm{m}$ and $1.7 \mu \mathrm{m}$ (Fig. $5 \mathrm{~b}$ ) and $5.2 \mu \mathrm{m}$ and $3 \mu \mathrm{m}$ (Fig. 5e), giving aspect ratios of 1.76 and 1.73 after longer etching, respectively. The aspect ratios evaluated from the etched structures depend on the etching period. These results suggest that marked etching occurred when Au-assisted chemical etching was performed for a long period. The aspect ratio of the hole aperture was the same after each stage of etching even though the arrangement of holes is different between Figs. 5a, 5b and 5d, 5e. This suggests that holes have aspect ratios that are independent of those of neighboring holes.

During metal-assisted chemical etching, the diffusion of positive holes (henceforth, " $\mathrm{h}^{+}$" is used to denote positive holes) away from the interface between the catalytic metal and substrate takes place. ${ }^{19}$ We consider that the $\mathrm{h}^{+}$diffused equally in all the crystallographic directions of the substrate, which means that metal-assisted chemical etching in the two-dimensional substrate proceeds in the radial direction of the substrate surface. At the same time, anisotropic chemical etching takes place in GaAs (100), which is induced by the substrate crystallography. For GaAs, these two types of etching enable us to obtain a high degree of freedom in the etching morphology, which cannot be achieved in the metal-assisted chemical etching of $\mathrm{Si}^{14}$

High controllability of Au-assisted chemical etching. - The effect of the noble-metal species on the metal-assisted chemical etching of GaAs was also examined. Here etching was conducted in an etchant consisting of $5 \mathrm{~mol} \mathrm{dm}^{-3} \mathrm{HF}$ and $1 \mathrm{~mol} \mathrm{dm}^{-3} \mathrm{H}_{2} \mathrm{O}_{2}$ for $60 \mathrm{~s}$ using $\mathrm{Au}$ (Fig. 6a) and Pt-Pd (Fig. 6b) as catalysts. Although the mechanism of metal-assisted chemical etching is identical regardless of the metal species, the morphology of the hole structures formed by Au-assisted chemical etching is more anisotropic than that of the structures formed by Pt-Pd-assisted chemical etching.

Since the depths of the structures were $\sim 1.2 \mu \mathrm{m}$ for $\mathrm{Au}$ and $\sim 1.4 \mu \mathrm{m}$ for Pt-Pd, the use of Pt-Pd resulted in slightly faster etching than that of $\mathrm{Au}$. On the basis of empirical results, we consider that the work function of the noble metal used as an etching catalyst affects the rate of metal-assisted chemical etching. In the metal-assisted chemical etching of $\mathrm{Si},{ }^{14}$ higher etching rate is generally obtained by using a catalyst with a large work function. Chourou et al. ${ }^{20}$ discussed the rate of formation of porous $\mathrm{Si}$ in the etching of $\mathrm{p}$-Si decorated by noble-metal particles as catalysts. In their study, they discussed the etching rate from the standpoints of the work function, the potential barrier $\left(\phi_{\mathrm{M}}\right)$ between the valence band edge of bulk Si and the $\mathrm{p}$-Si/metal interface, and $\mathrm{h}^{+}$injection toward the $\mathrm{p}$-Si/metal interface.

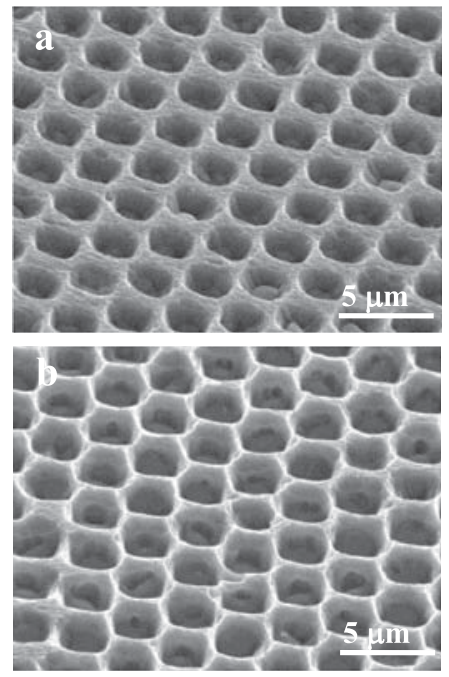

Figure 6. Hole arrays fabricated by (a) $\mathrm{Au}$ - and (b) Pt-Pd-assisted chemical etching for $60 \mathrm{~s}$ in $5 \mathrm{~mol} \mathrm{dm}^{-3} \mathrm{HF}$ and $1 \mathrm{~mol} \mathrm{dm}^{-3} \mathrm{H}_{2} \mathrm{O}_{2}$. The images were taken at $45^{\circ}$ to the surface.

According to their study, a low $\phi_{\mathrm{M}}$ value, which means that a metal has a relatively large work function, leads to increased $\mathrm{h}^{+}$injection toward the $\mathrm{p}-\mathrm{Si} / \mathrm{metal}$ interface. Conversely, a metal with a relatively small work function has a high $\phi_{\mathrm{M}}$ value. This suggests that it is difficult to inject $\mathrm{h}^{+}$into the $\mathrm{p}$-Si/metal interface. The amount of $\mathrm{h}^{+}$ supplied to the interface, which is strongly correlated to the value of $\phi_{\mathrm{M}}$ and the work function, mainly determines the rate of formation of porous $\mathrm{Si}$, the etching rate of $\mathrm{Si}$. The rapid etching of $\mathrm{Si}$ is realized when a sufficient amount of $\mathrm{h}^{+}$are injected toward the interface, where injection is promoted by the use of a metal catalyst with a large work function. On the basis of Ref. 20 and previously reported work function values, the etching rates of hole arrays catalyzed by $\mathrm{Au}$ and Pt-Pd in the present study showed qualitatively good agreement. The reported work functions are 5.1-5.28 eV for $\mathrm{Au},{ }^{21,22} 5.65 \mathrm{eV}$ for $\mathrm{Pt}^{21}$ and $5.12-5.55 \mathrm{eV}^{21,22}$ for Pd. These values indicate that metalassisted chemical etching using $\mathrm{Au}$ may be somewhat slower than that using Pt-Pd. Since the etching rate in Pt-Pd-assisted chemical etching is high, the chemical etching of the substrate during metalassisted chemical etching should be less anisotropic than that in the case of Au-assisted chemical etching. Therefore, only elliptical hole structures were obtained in Pt-Pd-assisted chemical etching. On the other hand, the high degree of freedom in the etching morphology, enabling the formation of rectangular, circular, and parallelogramshaped holes, resulted from the combination of Au-assisted chemical etching and anisotropic chemical etching.

Highly ordered InAs nanodot patterns can be applied to optical devices. To obtain such dot patterns, single InAs nanodots can be grown in single GaAs nanoholes. In such a case, a GaAs hole array is used as the framework for InAs growth; thus, the formation of well-ordered GaAs hole arrays with holes of a homogeneous size and sufficient regularity of order is crucial. Furthermore, the production of CNTs, considered to be one of the post-Si semiconductors, by various synthesis routes has been studied. In the presence of $\mathrm{Ag}$ nanoparticles, CNTs have been grown onto the particles through the direct reduction of a $\mathrm{C}$ source, ${ }^{23}$ where nanosize $\mathrm{Ag}$ acted as a catalyst for the selective growth of CNTs. The growth of CNTs on Au nanoparticle catalysts has also been reported. ${ }^{24} \mathrm{CNT}$-semiconductor hybrid systems exhibit novel properties that are generated by the combined properties of low-dimensional conductors and semiconductors. On the basis of this motivation, the site-selective growth of CNTs on a GaAs substrate using metal catalysts was attempted with the aim of realizing nanoscale electronic devices. ${ }^{25}$ Considering the above background and current industrial requirements, metal-assisted chemical etching is a promising technique for the flexible surface design of 
semiconductor substrates. ${ }^{26}$ Noble-metal-embedded GaAs hole arrays and/or hole arrays constructed using other III-V semiconductors are expected to be used in future multifunctional semiconductor devices.

\section{Conclusions}

Through the controlled Au-assisted chemical etching of n-GaAs (100), well-ordered hole arrays were formed. By changing the etching time, the concentration of HF in the etchant, and the metal species used as a catalyst, the morphology of hole arrays was sensitively altered, indicating the high potential of this method for the surface design of n-GaAs (100). GaAs (100) exhibited different etching rates in different crystallographic directions, thereby markedly anisotropic etching was observed. Consequently, various surface morphologies after etching were generated by the combined effects of anisotropic etching of the substrate and Au-assisted chemical etching.

The fabrication of porous semiconductors with a metal embedded at the bottom of each pore by a dry etching process is more complicated than the present process. Therefore, the downsizing of present structures obtained by the present technique is of great interest from the standpoints of producing a framework for the growth of nanotubes and/or nanorods and applications to functional electronic devices. The semiconductor nano/micro structures fabricated by the current metalassisted chemical etching method have expected to apply in the fields of solar energy conversion, ${ }^{27-32}$ thermal power conversion, ${ }^{33}$ and energy storage ${ }^{34}$ as chemical and biological sensors, ${ }^{35,36}$ and biomimic superhydrophobicity. ${ }^{37-39}$

\section{Acknowledgments}

This work was partly financially supported by a grant-in-Aid for Scientific Research (A) no. 20241026 from the Japan Society for the Promotion of Science. We also acknowledge the Strategic Research Foundation grant-aided Project: for Private Universities matching fund subsidy from the Ministry of Education, Culture, Sports, Science and Technology of Japan.

\section{References}

1. V. Lehmann and H. Föll, J. Electrochem. Soc., 137, 653 (1990).

2. A. G. Cullis, L. T. Canham, and P. D. J. Calcott, J. Appl. Phys., 82, 909 (1997).

3. S. Iijima, Nature, 354, 56 (1991).
4. P. Hoyer, Langmuir 12, 1411 (1996)

5. L. T. Canham, Appl. Phys. Lett., 57, 1046 (1990).

6. V. Lehmann and U. Gösele, Appl. Phys. Lett., 58, 856 (1991)

7. J. Bardwell, J. Webb, H. Tang, J. Fraser, and S. Moisa, J. Appl. Phys., 89, 4142 (2001).

8. X. Li, Y.-W. Kim, P. W. Bohn, and I. Adesida, Appl. Phys. Lett., 80, 980 (2002).

9. X. Y. Guo, T. L. Williamson, and P. W. Bohn, Solid State Commun., 140, 159 (2006).

10. L. Beji, L. Sfaxi, B. Ismail, S. Zghal, F. Hassen, and H. Maaref, Microelectron. J., 34, 969 (2003)

11. X. Li and P. W. Bohn, Appl. Phys. Lett., 77, 2572 (2000).

12. T. Hadjersi, N. Gabouze, A. Ababou, M. Boumaour, W. Chergui, H. Cheraga, S. Belhouse, and A. Djeghri, Mater. Sci. Forum, 480-481, 139 (2005).

13. H. W. Deckman and J. H. Dunsmuir, Appl. Phys. Lett., 41, 377 (1982).

14. H. Asoh, F. Arai, and S. Ono, Electrochim. Acta, 54, 5142 (2009).

15. S. Sakamoto, L. Philippe, M. Bechelany, J. Michler, H. Asoh, and S. Ono, Nanotechnology, 19, 405304 (2008).

16. Y. Yasukawa, H. Asoh, and S. Ono, J. Electrochem. Soc., 156, H777 (2009).

17. Y. Yasukawa, H. Asoh, and S. Ono, Jpn. J. Appl. Phys., 49, 116502 (2010).

18. H. Asoh, F. Arai, K. Uchibori, and S. Ono, Appl. Phys. Express, 1, 067003/1 (2008).

19. K. Tsujino and M. Matsumura, Electrochem. Solid-State Lett., 8, C193 (2005)

20. M. L. Chourou, K. Fukami, T. Sakka, S. Virtanen, and Y. H. Ogata, Electrochim. Acta, 55, 903 (2010).

21. D. E. Eastman, Phys. Rev. B, 2, 1 (1970).

22. B. E. Nieuwenhuys, R. Bouwman, and W. H. M. Sachtler, Thin Solid Films, 21, 51 (1974).

23. Z. Kang, E. Wang, S. Lian, L. Gao, M. Jiang, C. Hu, and L. Xu, Nanotechnology, 15 490 (2004).

24. S. Y. Lee, M. Yamada, and M. Miyake, Carbon, 43, 2654 (2005)

25. R. Engel-Herbert, Y. Takagaki, and T. Hesjedal, Mater. Lett., 61, 4631 (2007).

26. Z. Huang, N. Geyer, P. Werner, J. de Boor, and U. Gösele, Adv. Mater, 23, 285 (2011).

27. K. Q. Peng, Y. Xu, Y. Wu, Y. J. Yan, S. T. Lee, and J. Zhu, Small, 1, 1062 (2005).

28. H. Fang, X. D. Li, S. Song, Y. Xu, and J. Zhu, Nanotechnology, 19, 255703 (2008).

29. E. C. Garnett and P. D. Yang, J. Am. Chem. Soc, 130, 9224 (2008).

30. K. Q. Peng, X. Wang, and S. T. Lee, Appl. Phys. Lett., 92, 163103 (2008)

31. Y. J. Hwang, A. Boukai, and P. D. Yang, Nano Lett., 9, 410 (2009).

32. V. Sivakov, G. Andra, A. Gawlik, A. Berger, J. Plentz, F. Falk, and S. H. Christiansen, Nano Lett., 9, 1549 (2009).

33. A. I. Hochbaum, R. K. Chen, R. D. Delgado, W. J. Liang, E. C. Garnett, M. Najarian, A. Majumdar, and P. D. Yang, Nature, 451, 163 (2008).

34. K. Peng, J. Jie, W. Zhang, and S. T. Lee, Appl. Phys. Lett., 93, 033105 (2008).

35. B. H. Zhang, H. S. Wang, L. H. Lu, K. L. Ai, G. Zhang, and X. L. Cheng, Adv. Funct. Mater, 18, 2348 (2008).

36. M. L. Zhang, C. Q. Yi, X. Fan, K. Q. Peng, N. B. Wong, M. S. Yang, R. Q. Zhang, and S. T. Lee, Appl. Phys. Lett., 92, 043116 (2008).

37. M. W. Cao, X. Y. Song, J. Zhai, J. B. Wang, and Y. L. Wang, J. Phys. Chem. B, 110, 13072 (2006)

38. F. Shi, Y. Y. Song, H. Niu, X. H. Xia, Z. Q. Wang, and X. Zhang, Chem. Mater, 18, 1365 (2006)

39. Y. Xiu, L. Zhu, D. W. Hess, and C. P. Wong, Nano Lett., 7, 3388 (2007). 